\title{
The Possible Role of Bifidobacterium longum BB536 and Lactobacillus rhamnosus HN001 on Locomotor Activity and Oxidative Stress in a Rotenone-Induced Zebrafish Model of Parkinson's Disease
}

\author{
Ovidiu-Dumitru Ilie ${ }^{1},{ }^{1}$ Emanuela Paduraru, ${ }^{2}$ Madalina-Andreea Robea, ${ }^{1}$ \\ Ioana-Miruna Balmus, ${ }^{3}$ Roxana Jijie, ${ }^{3}$ Mircea Nicoara, ${ }^{1}$ Alin Ciobica ${ }^{(D)},{ }^{1}$ \\ Ilinca-Bianca Nita, ${ }^{4}$ Romeo Dobrin $\mathbb{D}^{5},{ }^{5}$ and Bogdan Doroftei ${ }^{4}$ \\ ${ }^{1}$ Department of Biology, Faculty of Biology, "Alexandru Ioan Cuza” University, Carol I Avenue, No 20A, 700505 Iasi, Romania \\ ${ }^{2}$ Faculty of Geography and Geology, "Alexandru Ioan Cuza" University, Carol I Avenue, No 20A, 700505 Iasi, Romania \\ ${ }^{3}$ Department of Exact and Natural Sciences, Institute of Interdisciplinary Research, "Alexandru Ioan Cuza" University, \\ Carol I Avenue, No 11, 700506 Iasi, Romania \\ ${ }^{4}$ Faculty of Medicine, University of Medicine and Pharmacy "Grigore T. Popa", University Street, No 16, 700115 Iasi, Romania \\ ${ }^{5}$ Department of Psychiatry, Faculty of Medicine, University of Medicine and Pharmacy "Grigore T. Popa", University Street, No 16, \\ 700115 Iasi, Romania
}

Correspondence should be addressed to Romeo Dobrin; romeodobrin2002@gmail.com

Received 2 September 2021; Accepted 13 September 2021; Published 14 October 2021

Academic Editor: Jolanta Czuczejko

Copyright (c) 2021 Ovidiu-Dumitru Ilie et al. This is an open access article distributed under the Creative Commons Attribution License, which permits unrestricted use, distribution, and reproduction in any medium, provided the original work is properly cited.

Background. As every organ within the body, the brain is also extremely susceptible to a plethora of noxious agents that change its chemistry. One component frequently found in current products against harmful species to crops is rotenone whose effect under prolonged exposure has been demonstrated to cause neurodegenerative disorders such as Parkinson's disease. The latest reports have indeed revealed that rotenone promotes Parkinson's in humans, but studies aiming to show congruent effects in zebrafish (Danio rerio) are lacking. Material and Methods. In this context, the aim of the present study was to demonstrate how chronic administration of rotenone for 3 weeks impairs the locomotor activity and sociability and induces oxidative stress in zebrafish. Results. There were no statistically significant differences following the analysis of their social interaction and locomotor tests $(p>0.05)$. However, several exceptions have been noted in the control, rotenone, and probiotics groups when we compared their locomotor activity during the pretreatment and treatment interval $(p<0.05)$. We further assessed the role of rotenone in disturbing the detoxifying system as represented by three enzymes known as superoxide dismutase (SOD), glutathione peroxidase (GPx), and malondialdehyde (MDA). Despite the fact that there were no statistically significant changes within SOD and GPx levels between the control group and rotenone, probiotics, and rotenone + probiotics $(p>0.05)$, relevant changes have been observed between the analyzed groups $(p<0.05$ and $p<0.005$, respectively). On the other hand, significant differences $(p<0.05)$ have been observed for MDA when we analyzed the data between the control group and the other three groups. Conclusions. Our results suggest that rotenone can be successfully used to trigger Parkinson's disease-related symptomatology in zebrafish. 


\section{Introduction}

Parkinson's disease (PD) is the second most common neurodegenerative disorder after Alzheimer's disease (AD) and is expected to advance by 2050 . According to the latest published statistics, the incidence and prevalence fluctuate around 4.5-21, respectively, 18-328 cases at 100,000 individuals per year ( $\sim 10$ million worldwide). Although of onset is in the range of the fourth and seventh decades of life, the existing evidence refutes this hypothesis [1].

Mechanistically speaking, PD is characterized by a constant loss of dopaminergic and cholinergic neurons from the substantia nigra pars compacta $(\mathrm{SNpc})$ and posterior motor nucleus of the vagus. Furthermore, it is characterized by the continuous storage and aggregation of the $\alpha$-synuclein within the central nervous system (CNS) [2].

Despite best efforts, the etiology of PD remains obscure, recent studies revealing that $\mathrm{PD}$ actually possesses a multifactorial substrate, reflected by major phenotypic changes. Specifically, the clinical signs exhibited by patients are grouped under the umbrella of the Parkinsonism spectrum and include essential tremor, stiffness, bradykinesia, and impaired postural balance [2].

Regarding its origin, PD has been shown to be caused by exposure to exogenous stressors (pesticides, heavy metals, or illicit substances) [3], either by abnormal gene expression, with or without family aggregation. By default, these determinants may gradually induce autosomal dominant PD or autosomal recessive juvenile PD [4]. However, people diagnosed and treated with antipsychotics are prone to significant side effects, a phenomenon known as drug-induced Parkinson's syndrome (PID) [5].

Cumulatively, it can be argued that PD is gradually triggered by various factors, but there are also situations in which the cause is idiopathic. Here was another long-time discussion due to the clinical heterogeneity and overlap between PD dementia, Lewy body dementia, and other types, which is why the nomenclature has been constantly reevaluated [6].

One common and usually underexplored feature of PD patients, yet crucial, is the role of anxiety [7] and depression [8] as promoters and/or pointers of PD. Analogous for healthy patients exposed to prolonged stress states as a risk group. The associated changes of the brain's chemistry have been extensively discussed from both perspectives [9, 10], and especially the petulant and abnormal oxidative stress (OS) that disrupts the physiological status of the redox potential [11]. The missing link of this puzzle could be the gut microbiota due to its ability to shape humans behavior and development, regardless of age, sex, and health status [12].

The most powerful vehicle used in clinical practice to diminish PD-related symptomatology and the associated comorbidities causative or not are probiotics [13]. These are live microorganisms known to have a helpful role in improving digestive function $[14,15]$. Their implication in PD was often suggested in the last years [13, 16, 17]. Along this, there are not too many studies to sustain their importance in the PD diet but, until now, the evidence strongly supports probiotics [18]. It has been demonstrated in three distinct randomized controlled trials that probiotics have the capacity to ameliorate all related-gastrointestinal deficiencies (constipation) in patients compared with the placebo. More precisely, the authors administered between 1 and 3 month strains of Streptococcus, Enterococcus, and especially Bifidobacterium and Lactobacillus and observed an improvement in life's quality, changes in fecal calprotectin, and less bowel deficiencies. However, the results following Unified Parkinson's Disease Rating Scale remained low despite the therapy [18-20].

Various animal models are being used in modeling PD disorder in order to observe the mechanisms or even possible find new therapeutic interventions in ameliorating and/or treating it. The successful usage of Danio rerio as a model for neurologic disorders is due to its main advantages such as physiological homology to human individuals, short time of replication, and low cost of breeding as compared to other experimental models, and it is also suitable for drug development studies [21]. Its implication in PD modeling was described and published in many reports over the years [22-27].

The pesticide rotenone was often used for inducing PD symptoms in rodents but also there are several studies which assessed rotenone's impact on zebrafish [28-31]. Rotenone was for a long time utilized in several commercialized insecticides, pesticides, or piscicides [24]. Beside behavioral aspects, rotenone is able to intervene in the OS balance by influencing enzymes level responsible with antioxidant role [32].

Having as support all these data, the aim of this study was to determine if probiotics brought a beneficial role in reducing the oxidative status and reestablishment potential of the motor impairment in a zebrafish PD model.

\section{Material and Methods}

2.1. Animal Husbandry. A total of sixty adults (6-8 months old) wild-type (WT) zebrafish (Danio rerio) were purchased from a local breeder. The fish were accommodated three weeks to laboratory conditions and kept in a $90 \mathrm{~L}$ recirculating dechlorinated water aquarium. Both aquariums (housing and experimental) respected the standard parameters as $26 \pm 2{ }^{\circ} \mathrm{C}$ temperature, $\mathrm{pH} 7.5$, with a $14 \mathrm{~h}$ light $/ 10 \mathrm{~h}$ night cycle [33]. Water was changed daily in the experimental tanks. The adults were fed twice per day with TetraMin Flakes.

2.2. Ethical Approval. This experiment was performed by respecting the EU Commission Recommendation (2007), Directive 2010/63/EU of the European Parliament, and the Council of 22 September 2010 regarding protection, accommodation, and care of animals for scientific/experimental purposes $[34,35]$. The current protocol was approved by the Ethical Committee of the Faculty of Biology, "Alexandru Ioan Cuza" University of Iasi with the registration number 11/25.05.2021.

\subsection{Chemicals}

2.3.1. Rotenone. Rotenone $\left(\mathrm{C}_{23} \mathrm{H}_{22} \mathrm{O}_{6}\right)$ was purchased from Toronto Research Chemicals, North York, Canada (Cat\# 
R700580), under a white powder that has been dissolved in distilled water until we reached a $2 \mu \mathrm{g} \mathrm{L}^{-1}$ concentration that was further administered daily for 21 days [30, 36].

2.3.2. Probiotics. Zircombi (ALFASIGMAS.p.A.) is a dedicated food supplement for people, having the appearance of a white powder that was purchased from a local pharmacy. It contains two strains-Bifidobacterium longum BB536- $4 \times 10^{9} \mathrm{CFU}(150 \mathrm{mg})$ and Lactobacillus rhamnosus HN001-1 $\times 10^{9} \mathrm{CFU}(25 \mathrm{mg})$ and Vit $\mathrm{B}_{6}-1.4 \mathrm{mg}$. We dissolved this mixture in $1 / 4$ water using a $100 \mathrm{ml}$ rated balloon being administered half an hour before routine feeding in rearing water to ensure the ingestion as indicated by Valcarce et al. and obtaining as follows: $3 \mathrm{mg} \mathrm{L}^{-1}$ BB536, $0.5 \mathrm{mg} \mathrm{L}^{-1}$ HN001, and $0.02 \mathrm{mg} \mathrm{L}^{-1}$ vit. $\mathrm{B}_{6}$ [37].

2.4. Design Protocol. After the accommodation period was over, they were subsequently divided into four equal groups $(n=15)$ and placed in small $10 \mathrm{~L}$ tanks for another two days in order to adapt to the new environment. Afterward, we assessed their swimming performance and sociability to determine whether or not are differences between pretreatment and treatment period. The groups were as follows: group 1 (control), group 2 (probiotics), group 3 (rotenone), and group 4 (rotenone + probiotics). The substances were daily renewed after water changing. The entire study lasted 21 days, and at the end of the experimental period, fishes were killed in water with ice at a temperature under $5^{\circ} \mathrm{C}$ [38].

2.5. Behavioral Assessment. The social interaction test was performed daily to evaluate the changes induced by rotenone and the possible behavior benefic role of probiotics. For this, we used a modified cross maze labyrinth closed by a slit and transformed into a T maze $(10 \mathrm{~h} \times 50 \mathrm{l} \times 50 \mathrm{w} \mathrm{cm})$ filled with system water $(5 \mathrm{~cm})[39,40]$. The social stimulus was placed in the left arm of the T maze (Figure 1). The locomotor activity test was performed in the same maze. Once introduced, each fish was let to acclimate to the new conditions for half a minute. Movement was registered with a professional camera placed above the experimental chamber over a period of 240 seconds. Parameters were analyzed using EthoVision XT 11.5 software (Noldus Information Technology, Wageningen, The Netherlands). We focused on 3 specific parameters such as the total distance swam $(\mathrm{cm})$, velocity $\left(\mathrm{cm} \mathrm{s}^{-1}\right)$, and active status (s) for locomotor activity test and the time spent in the left arm (s) for social interaction test.

2.6. Oxidative Stress Measurement and Sample Preparation. Superoxide dismutase determination kit (SOD, 19160-1KTF), Glutathione Peroxidase Cellular Activity Assay Kit (GPx, CGP1-1KT), and Protein Quantification Kit-Rapid (51254-KT) were purchased from Merck, Germany. All the analyses were performed according to the manufacturer's instructions. Malondialdehyde (MDA) levels were assessed by thiobarbituric acid-reactive assay following a preestablished work protocol [41].

At the end of chronic exposure period, each fish was individually well homogenized in a 10 volume of ice-cold saline $(0.90 \% \mathrm{NaCl})$. Each sample was centrifuged at $5500 \mathrm{rpm}$ for $10 \mathrm{~min}$ in accordance with the already estab-
The locomotor activity test

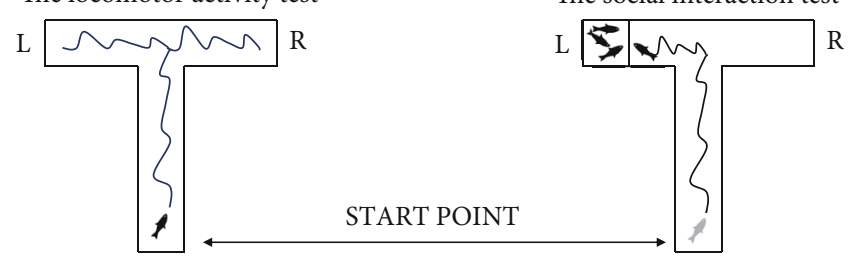

FIgURE 1: The experimental designs of zebrafish behavioral testing adapted to T-maze.

lished protocols by Jin et al. [42] and $\mathrm{Ni}$ et al. [43]. The supernatant obtained was aliquoted into $2 \mathrm{ml}$ Eppendorf tubes for the subsequent determination of oxidative biomarkers levels. A spectrophotometer at distinct wavelengths (Specord 210 Plus producer Analytik Jena, Germany) was used to assess each enzyme activity.

2.7. Statistical Analysis. Through the Shapiro-Wilk test, we first analyzed the normality and distribution of the data, Microsoft Excel 2010 being used for editing, sorting, and coding of the raw data. The data was then exported into OriginPro v.9.3 (2016) software (OriginLab Corporation, Northampton, MA, USA). Thereafter, we used one-way ANOVA and Tukey HSD test to verify and certify whether or not there are significant different variances among investigated parameters from the start until endpoint $[39,44]$. For the abovementioned behavioral parameters, data are presented as average \pm SEM. For OS, we calculated the variance between the control and the experimental groups performing multiple comparisons; data expressed as average \pm SEM. $p<0.05$ was regarded as statistically significant.

\section{Results}

3.1. The Impact of Rotenone and Probiotics on Zebrafish Swimming Performance. According to our swimming performance, parameters as the total distance swam and the average velocity showed no pronounced effects of rotenone or probiotics administration after 21 days. Regarding the total distance swam, all the experimental groups recorded several changes between pretreatment and treatment period. The control group showed several picks of activity during experimental period compared to the pretreatment days as: D_10 $(1240.03 \pm 169.9 \mathrm{~cm}, \quad p=0.01$ Tukey, ANOVA $), \quad$ D_13 $(1209.58 \pm 71.7 \mathrm{~cm}, p=0.02$ Tukey, ANOVA), and D_15 $(1220.13 \pm 104.2 \mathrm{~cm}, p=0.02$ Tukey, ANOVA) vs. $620.7 \pm$ $127.8 \mathrm{~cm}$. The second group exposed to rotenone did not present significant changes during the treatment excepting D_6 $(1369.2 \pm 255.8 \mathrm{~cm}, p=0.008$ Tukey, ANOVA $)$ and D_14 $(1642.9 \pm 165.1 \mathrm{~cm}, p=0.002$ Tukey, ANOVA $)$.

The third group treated with probiotics showed an increase of distance swam in the first days of the treatment with a highest value for the D_5 $(1626.4 \pm 138.2 \mathrm{~cm}, p=$ 0.006 Tukey, ANOVA). After 1 week of administration, the trend was composed from ups and downs as it can be seen in Figure 2. When rotenone and probiotics were administrated together, the activity of this parameter did not record important modifications ( $p>0.05$ ANOVA). 


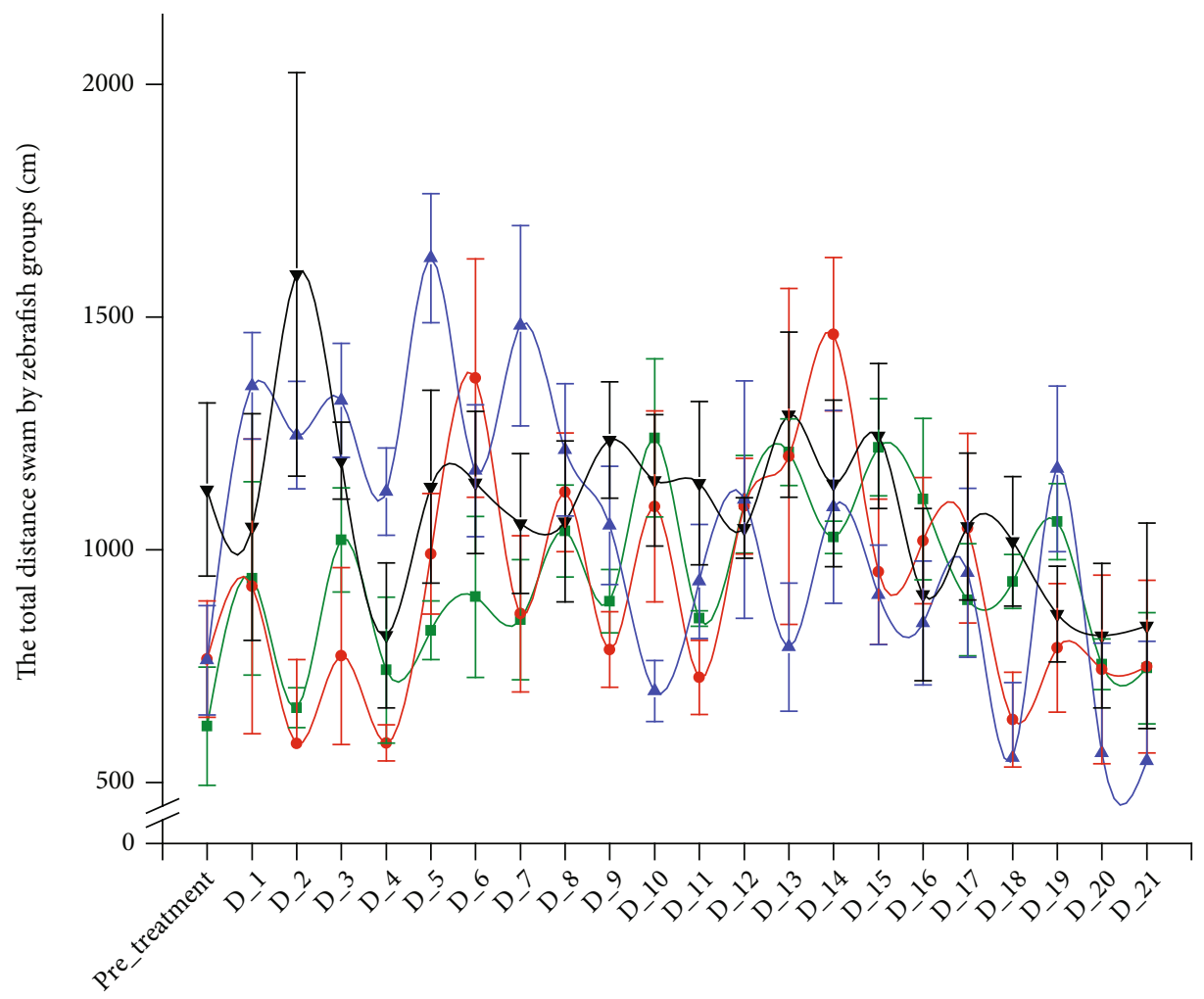

Time of exposure (days)

$$
\begin{aligned}
& \because \text { Control } \\
& \because \text { Rot }
\end{aligned}
$$$$
\rightarrow \text { Probio }
$$$$
\rightarrow \text { Rot }+ \text { Probio }
$$

FIGURE 2: The total distance swam by experimental groups during locomotor activity test $(n=15)$. Green: control group, red: rotenone $\left(2 \mu \mathrm{g} \mathrm{L}^{-1}\right)$, blue: probiotics $\left(3 \mathrm{mg} \mathrm{L}^{-1}\right.$ BB536, $0.5 \mathrm{mg} \mathrm{L}^{-1} \mathrm{HN001}$, and $0.02 \mathrm{mg} \mathrm{L}^{-1}$ vit. $\left.\mathrm{B}_{6}\right)$, black: rotenone $\left(2 \mu \mathrm{g} \mathrm{L}^{-1}\right)$ and probiotics $\left(3 \mathrm{mg} \mathrm{L}^{-1}\right.$ BB536, $0.5 \mathrm{mg} \mathrm{L}^{-1} \mathrm{HN} 001$, and $0.02 \mathrm{mg} \mathrm{L}^{-1}$ vit. $\mathrm{B}_{6}$ ). The groups mean was compared to pretreatment days mean, and the results were represented as average \pm SEM.

No significant changes were observed for control, rotenone, and mixture group regarding the velocity parameter $(p>0.05$ ANOVA). The trend was similar for the probiotic group excepting the short boost of hyperactivity in D_5 $(6.77 \pm 0.57 \mathrm{~cm} / \mathrm{s}, p=0.005$ Tukey, ANOVA) compared to the pretreatment: $3.18 \pm 0.42 \mathrm{~cm} / \mathrm{s}$ (Figure 3).

We also determined the active status which measures the time spent by fish being active during the session. In the pretreatment days, fish from the control group showed a decrease in time moving, but its activity during the whole experimental period was following a constant trend. Fish exposed to rotenone recorded increases in time spent moving as D_6 (224.8 $\pm 10.8 \mathrm{~s}, p=0.03$ Tukey, ANOVA), D_8 $(228.8 \pm 5.22 \mathrm{~s}, p=0.02$ Tukey, ANOVA), D_12 (227.2 \pm $3.52 \mathrm{~s}, p=0.02$ Tukey, ANOVA), D_14 (236.3 $\pm 1.50 \mathrm{~s}, p=$ 0.007 Tukey, ANOVA), D_16 (232.1 $\pm 3.78 \mathrm{~s}, p=0.01$ Tukey, ANOVA), and D_17 (223.5 $\pm 4.55 \mathrm{~s}, p=0.04$ Tukey, ANOVA) in comparison to $139.8 \pm 12.7 \mathrm{~s}$ of pretreatment time. Regarding the activity for the third group treated with probiotics, its activity was similar with those observed for the abovementioned parameters and increases in the first part of the administration and then ups and downs. However, there was no statistically significant difference for the mixture group ( $p>0.05$ ANOVA) (Figure 4 ).
3.2. No Effect of Rotenone and Probiotic Administration on Zebrafish Sociability. No significant changes were observed for the control and rotenone group ( $p>0.05$ ANOVA). The single administration of rotenone or probiotics did not have any effect on zebrafish sociability after 21 days of exposure as it can be seen in Figure 5. Moreover, a similar effect was also observed for the last group excepting D_19 when time spent next to the group increased considerably compared to pretreatment period $(182.5 \pm 16.5 \mathrm{~s}$ vs. $56.2 \pm 19.8 \mathrm{~s}, p=0.01$ Tukey, ANOVA).

3.3. Oxidative Stress. While analyzing the data regarding OS after 21 days of rotenone administration in zebrafish individuals, we noted several changes (Figure 6). For SOD, there was no statistically significant difference between the control group and the other three $(p>0.05)$ but rather when we compared the rotenone group with probiotics $(p=0.014)$ and probiotics + rotenone $(p=0.011)$. SOD level dropped in the rotenone-exposed group compared to the control group. Instead, the probiotics group showed an increase in SOD activity, result which was expected to obtain knowing the fact that probiotics can enhance SOD expression in living cells [45] and the differential response of each body depending on the organ investigated [46]. Meanwhile, the 


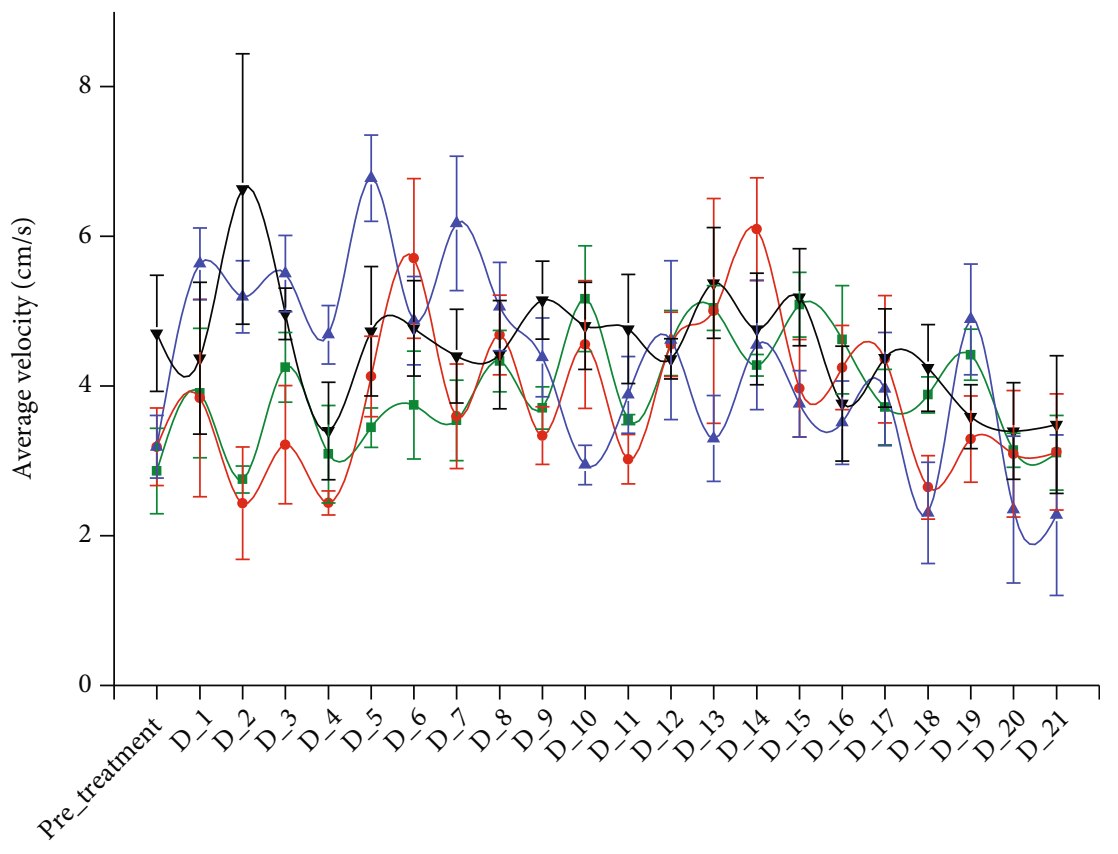

Time of exposure (days)

$$
- \text { Control }
$$$$
\begin{aligned}
& \longrightarrow \text { Probio } \\
& \longrightarrow \text { Rot }+ \text { Probio }
\end{aligned}
$$

FIGURE 3: The average velocity of the experimental groups recorded during locomotor activity test $(n=15)$. Green: control group, red: rotenone $\left(2 \mu \mathrm{g} \mathrm{L}^{-1}\right)$, blue: probiotics $\left(3 \mathrm{mg} \mathrm{L}^{-1} \mathrm{BB} 536,0.5 \mathrm{mg} \mathrm{L}^{-1} \mathrm{HN} 001\right.$, and $0.02 \mathrm{mg} \mathrm{L}^{-1}$ vit. B 6 ), black: rotenone $\left(2 \mu \mathrm{g} \mathrm{L}^{-1}\right)$ and probiotics ( $3 \mathrm{mg} \mathrm{L}^{-1} \mathrm{BB} 536,0.5 \mathrm{mg} \mathrm{L}^{-1} \mathrm{HN} 001$, and $0.02 \mathrm{mg} \mathrm{L}^{-1}$ vit. $\mathrm{B}_{6}$ ). The group mean was compared to pretreatment days mean, and the results were represented as average \pm SEM.

last group exposed to rotenone and probiotics had also an increase in SOD level but still with no considerable change compared to control.

In the case of GPx, analogous results were obtained since there were no statistically significant differences between the control group and the rest of the groups investigated $(p>0.05)$. On the other hand, there was a significant change of GPx level when compared rotenone group with probiotics $(p=0.001)$ and rotenone + probiotics $(p=0.004)$ groups. It is already known that OS is often reported as a risk factor in PD development [47-49]. SOD and GPx are considered to be the first line defense against free radicals [50] and a diet supplementation with Lactobacillus fermentum enhance the antioxidant system as reported by Wang et al. [51]. A similar pattern has been also observed for GPx despite the fact that no significant difference, whereas the level of SOD is compensatory increased. Based on these considerations, there is certainty of the existence of OS. The possible explanation is that Bifidobacterium longum BB536 and Lactobacillus rhamnosus HN001 modulate anxiety state in Danio rerio but at the same time promote a hyperlocomotor activity [52], hence, these abnormal levels of OS biomarkers.

Furthermore, the main marker of lipidic peroxidation, MDA, presented significant high levels $(p<0.05)$ in the control group as compared with probiotics $(p=0.033)$ and in contrast with rotenone and probiotics $(p=0.032)$. Also, there were significant changes in terms of MDA levels between the rotenone group and probiotics $(p=0.009)$ and rotenone + probiotics $(p=0.01)$ groups, respectively. Congruent with other studies, it appears that MDA is significantly increased after 30 days of $2 \mathrm{mg} \mathrm{L}^{-1}$ rotenone administration [46], Bifidobacterium longum BB536 being a reliable bacteria attributed to the reestablishment of the antioxidant system [53].

\section{Discussion}

A variety of reports have highlighted that fish, among other aquatic organisms, are deeply sensitive to pollutants and also to natural compounds used as pesticide. In our study, chronic administration of rotenone was performed in zebrafish adults, and its effect on behavior and OS was measured. We observed that administration of $2 \mu \mathrm{g} \mathrm{L} \mathrm{L}^{-1}$ rotenone had impacted the total distance travelled during test session presenting ups and downs compared to the pretreatment time. For example, Khotimah et al. [54] reported a decreased motility after 2 weeks of $5 \mu \mathrm{g} / \mathrm{L}$ rotenone administration. As already reported by Wang et al. [30] and our team, there were no significant differences in terms of locomotor impairment, thus, further highlighting the fact that PD triggering in Danio rerio is dependent on dosage and time of administration. These changes in locomotor activity can be a consequence of mitochondrial dysfunction which is responsible with ATP production or/and overproduction of free radicals. It was suggested that these abovementioned can lead to dopamine deficiency and apoptosis by the study 


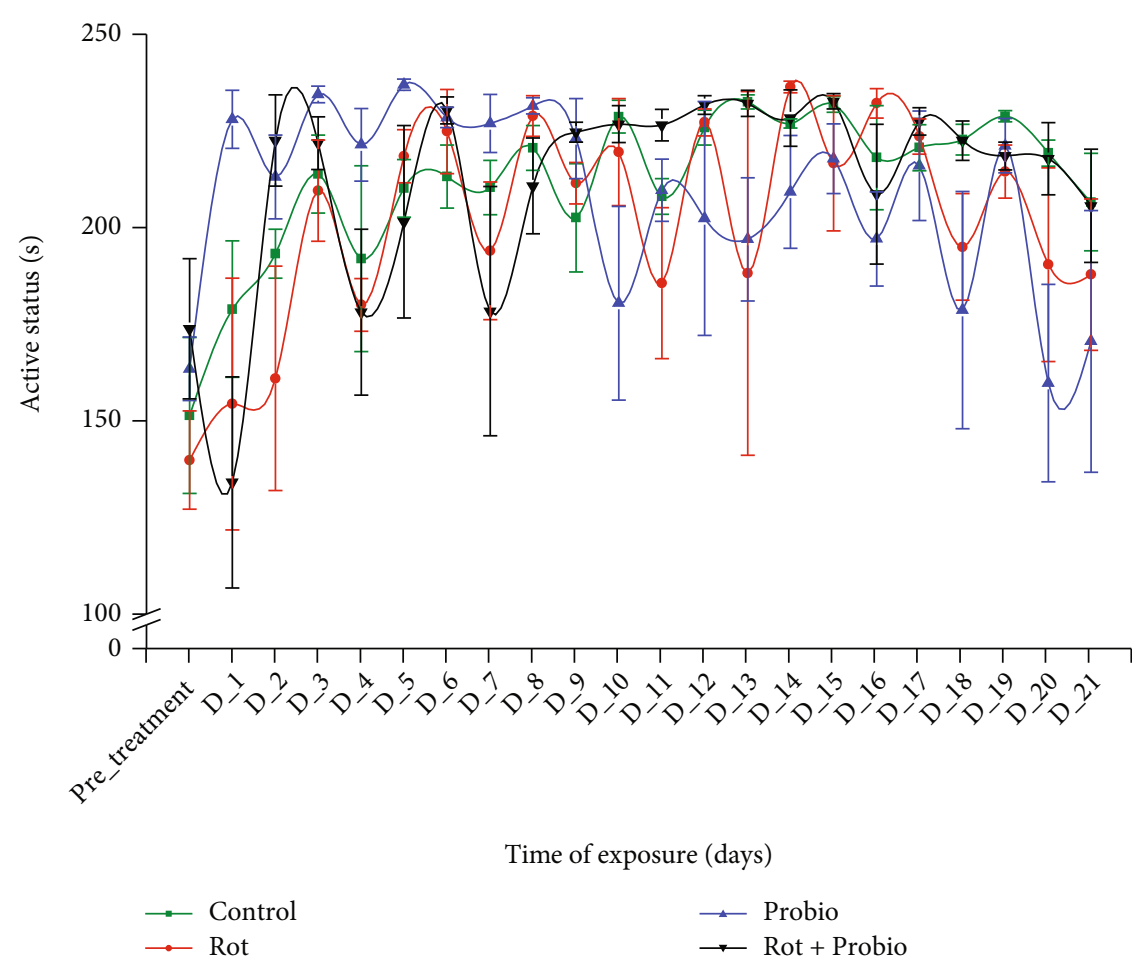

FIGURE 4: The active status of the experimental groups recorded during locomotor activity test $(n=15)$. Green: control group, red: rotenone $\left(2 \mu \mathrm{g} \mathrm{L}^{-1}\right)$, blue: probiotics $\left(3 \mathrm{mg} \mathrm{L}^{-1} \mathrm{BB} 536,0.5 \mathrm{mg} \mathrm{L}^{-1} \mathrm{HN} 001\right.$, and $0.02 \mathrm{mg} \mathrm{L}^{-1}$ vit. $\left.\mathrm{B}_{6}\right)$, black: rotenone $\left(2 \mu \mathrm{g} \mathrm{L}^{-1}\right)$ and probiotics $\left(3 \mathrm{mg} \mathrm{L}^{-1}\right.$ BB536, $0.5 \mathrm{mg} \mathrm{L}^{-1} \mathrm{HN001}$, and $0.02 \mathrm{mg} \mathrm{L}^{-1}$ vit. $\mathrm{B}_{6}$ ). The groups mean was compared to pretreatment days mean, and the results were represented as average \pm SEM.

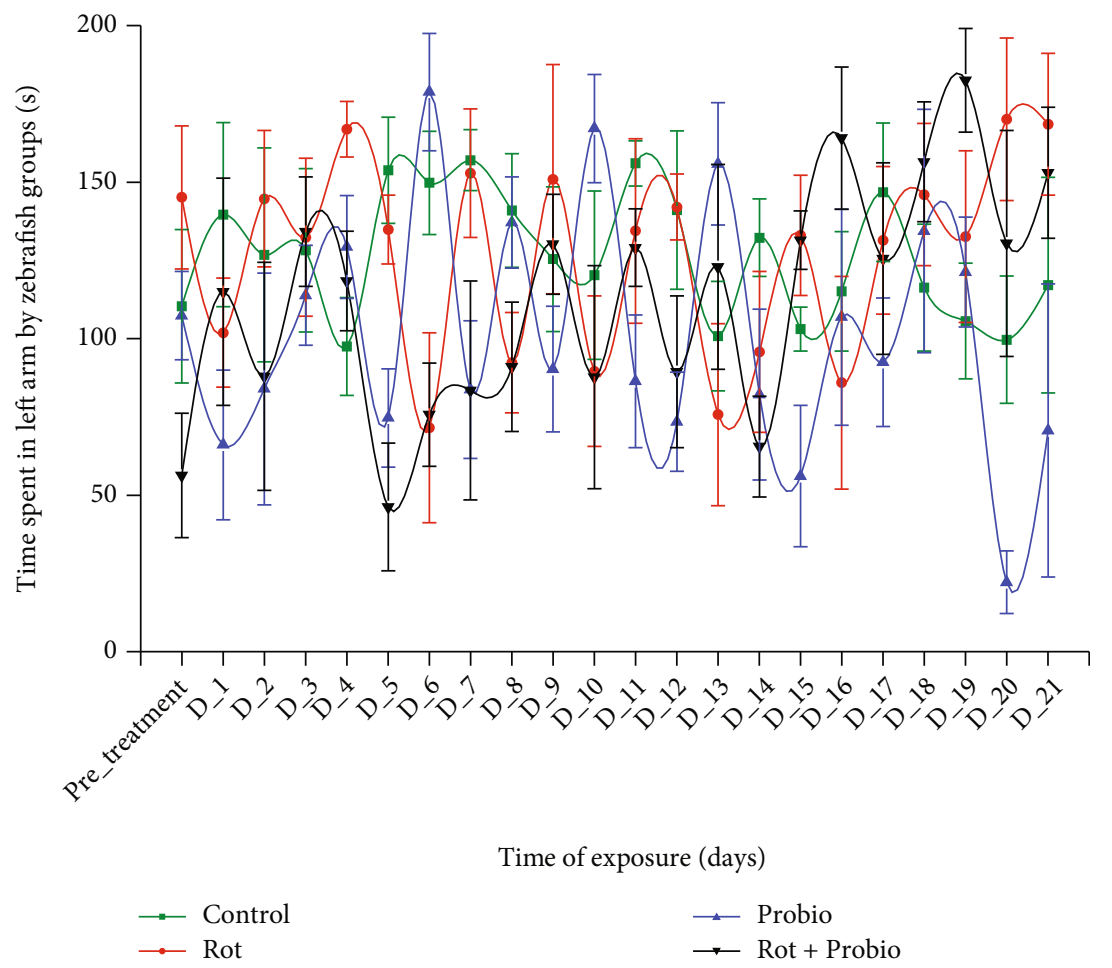

FIGURE 5: The time spent in the left arm by the experimental groups recorded during social interaction test $(n=15)$. Green: control group, red: rotenone $\left(2 \mu \mathrm{gL}^{-1}\right)$, blue: probiotics $\left(3 \mathrm{mgL}^{-1} \mathrm{BB} 536,0.5 \mathrm{mg} \mathrm{L}^{-1} \mathrm{HN} 001\right.$, and $0.02 \mathrm{mg} \mathrm{L}^{-1}$ vit. $\left.\mathrm{B}_{6}\right)$, black: rotenone $\left(2 \mu \mathrm{gL}^{-1}\right)$ and probiotics ( $3 \mathrm{mg} \mathrm{L}^{-1} \mathrm{BB} 536,0.5 \mathrm{mg} \mathrm{L}^{-1} \mathrm{HN} 001$, and $0.02 \mathrm{mg} \mathrm{L}^{-1}$ vit. $\mathrm{B}_{6}$ ). The groups mean was compared to pretreatment days mean, and the results were represented as average \pm SEM. 

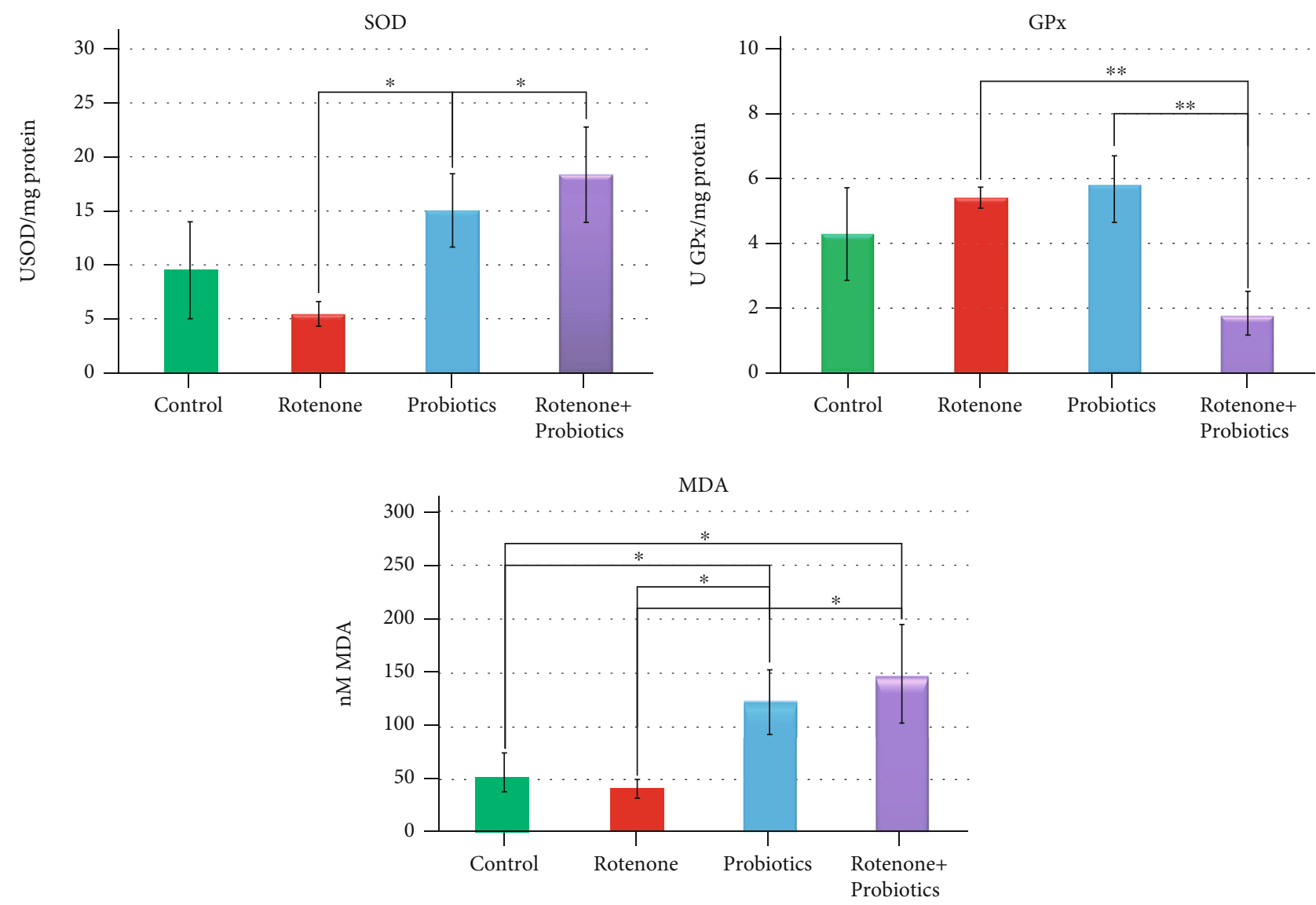

FIgURE 6: The activity of superoxide dismutase (SOD), glutathione peroxidase (GPx), and malondialdehyde (MDA) levels in zebrafish chronically exposed to rotenone and probiotics. Data were expressed as average \pm SEM $\left({ }^{*} p<0.05 ;{ }^{* *} p<0.005\right)$.

of Khotimah and his team in 2015 after chronic treatment of zebrafish with $5 \mu \mathrm{g} \mathrm{L}^{-1}$ rotenone for 28 days [54]. One year later Khatri and Juvekar also evaluated the influence of $5 \mu \mathrm{g} \mathrm{L}^{-1}$ rotenone on swimming behavior after 14 days of treatment [55].

When rotenone and probiotics were given together, this parameter did not show any significant changes. This could be an effect triggered by probiotic presence in the medium based on the third group results exposed only to probiotics where their activity was particularly increased in the first week of the treatment than the normal behavior recorded in the pretreatment.

It is interesting to observe that active status was different in the case of the second and forth group which received rotenone, respectively, rotenone and probiotics in the first day of treatment. Rotenone administration led to a hazardous zebrafish activity for all the period in comparison to the rotenone and probiotics group whose activity was adjusted after only 1 week of probiotic treatment.

To determine if rotenone has certain effects on zebrafish sociability the social interaction test was performed daily. According to our data, no significant impact on sociability was registered, fish manifesting contradictory behavior day after day. Even probiotic administration did not induce important advances in zebrafish social domain; it just sustains the normal activity. It was showed that the adminis- tration of probiotics together with oxytocin could have a positive influence on social domain in an ASD mouse model [56].

OS is a common risk factor often reported in PD pathogenesis $[48,57]$. Described as imbalance between antioxidants and oxidants, OS generates serious cellular damage which causes, mainly, neuronal degeneration [58]. In our study, we observed several changes in the antioxidant enzymes activity and an increased level of lipidic peroxidation. Treatment with $2 \mu \mathrm{gL}^{-1}$ rotenone for 21 days leads to a decrease in SOD level which was in agreement with Khan's study observations made on $\mathrm{PD}$ patients regarding OS markers [49]. On the other hand, SOD production was encouraged by probiotics. For example, diet supplementation with Lactobacillus fermentum could increase the level of SOD and GPx in pigs [51].

Exposing adult zebrafish to $2 \mathrm{mg} \mathrm{L}^{-1}$ rotenone for 30 days showed significant activity of MDA and decreased SOD level both intestines and brain for glutathione-S-transferase (GST) and catalase (CAT) level [46]. Neurodegeneration and OS have also been recorded after experimental exposure of human liver HepG2 cells to a range between 12.5 and $250 \mu \mathrm{M}$ rotenone for 24 hours [59]. If rotenone favors OS, probiotic presence can stimulate the antioxidant system as it was demonstrated in our study by high levels of SOD and GPX. 
Due to potentially antioxidant role, probiotics started to receive more attention, and since OS was linked to altered gut microbiota. The gut-brain axis is an important interaction which has been reported to be disrupted in PD individuals [60-62].

For example, Bhattaraiet al. and Dodiya and coauthors $[63,64]$ administered $10 \mathrm{mg} / \mathrm{kg} /$ day of rotenone for six and twelve weeks, respectively, and brought several solid arguments in this context. The administration for a period of 6 weeks led to a loss of tyrosine hydroxylase (TH) neurons in both conventionally raised mice and germ-free. In parallel, it resulted in a decrease in motor strength and coordination. The researchers concluded that chronic rotenone intake did not cause dysbiosis, rather a shift among bacterial load and increased in the first month and permeability in conventionally raised mice. On the other hand, it promoted elevated urinary cortisol, intestinal hyperpermeability, and a diminished abundance of lactic bacteria (Lactobacillus) in restraint stress mice by comparison with the control during the first month and a half. Moreover, rotenone alone without restraint stress disrupted the colonic expression of the tight junction protein ZO-1 and increased the protein level of $\alpha$-syn in the colon, N-tyrosine as a marker of OS, and myenteric plexus enteric glial cell-glial fibrillary acidic protein expression by comparing with the control group. In the restraint stress rotenone-induced was observed analogous changes as in the rotenone alone group with several exceptions; intestinal hyperpermeability, an abnormal expression of Occludin and Claudin1, and increased ratio of fecal Akkermania and endotoxemia. Nevertheless, an intraperitoneal injection of $2.75 \mathrm{mg} / \mathrm{kg}$ rotenone, 5 days per week for 1 month promoted GI dysfunctionalities like diarrhea and delayed gastric emptying. The subsequent microbiota analyses revealed alterations along the small intestine and colon, whereas histological examination indicates a mucosal thickening and goblet cell hyperplasia in the colon [65].

Nonetheless, Bifidobacterium longum BB536 and Lactobacillus rhamnosus HN001 are two probiotic strains that have been previously administered also in patients suffering from irritable bowel syndrome (IBS). Specifically, there was a statistically significant decrease $(p<0.0001)$ within two parameters regarding "abdominal pain" and "bloating" and overall severity of disease between IBS patients and placebo. The beneficial effects were more detectable in IBS-diarrhea patients as indicated by the Bristol scale $(p<0.00001)$ in contrast with placebo $(p=0.04)$, while in IBS-constipation, there was only a slight improvement $(p=0.06)$ [66]. Similar findings were made by another team in which they enrolled lactose intolerance patients vs. placebo. This time, the physical symptoms panel as indicated by "bloating" $(p=0.028)$ and "constipation" ( $p=0.045)$ were ameliorated in lactose intolerance patients as compared to placebo.

Another preliminary study revealed an increased load of both these species in pre- and postprandial individuals that persisted even after one month following the end of oral intake. At the phyla level, there was observed a significant decrease of Firmicutes, Proteobacteria as compared with the sample collected prior to the actual procedure. At the species level, the authors noted an increased abundance of several microorganisms including Blautia producta/wexlerae, Haemophilus ducrey, Akkermansia muciniphila, Roseburia faecis, and Ruminococcus gnavus during follow-up, followed by a reduction of Holdemania filiformis, Escherichia vulneris, Gemmiger formicilis, and Streptococcus sinensis [67].

This mixture has been also successfully administered in rat models. According to the results of Alsheraji et al., [53] Bifidobacterium longum BB536 reduced total cholesterol, very-low, low and high-density lipoprotein cholesterol, and atherosclerotic index amounts, concomitantly with that of liver-lipid deposition and adipocyte size. In the same study, they demonstrated that the plasma MDA level significantly decreased after the administration of Bifidobacterium longum BB536.

Bifidobacterium longum BB536 and Lactobacillus rhamnosus HN001, in combination and/or individually, possess the ability to inhibit the activity of the major Gramnegative strains, even opportunistic pathogenic entities such as Candida [68, 69]. Different teams of researchers have also revealed in several other occasions that rotenone causes a dysfunction of the rat's and mice's gastrointestinal microbiota as well. Precisely, there was observed a decrease of Bifidobacterium genus, as well as in Firmicutes and Bacteroides phyla $[70,71]$.

\section{Conclusions}

It can be concluded based on our results that rotenone is a potent toxic agent that can be successfully used to trigger PD-related symptomatology in Danio rerio. Furthermore, it can offer a pathway to improve our knowledge regarding the etiology of $\mathrm{PD}$, and these observations can be in the future further extrapolated to human individuals. Even though Bifidobacterium longum BB536 and Lactobacillus rhamnosus HN001 did not improve the locomotor activity, nor diminish oxidative status in zebrafish but rather induced a hyper-activity, our study offered a new perspective in contrast with the existing information.

\section{Data Availability}

The datasets used and analyzed during the current study are available from the corresponding author on reasonable request.

\section{Conflicts of Interest}

The authors declare no conflict of interest.

\section{References}

[1] E. R. Dorsey, A. Elbaz, E. Nichols et al., "Global, regional, and national burden of Parkinson's disease, 1990-2016: a systematic analysis for the global burden of disease study 2016," Lancet Neurology, vol. 17, no. 11, pp. 939-953, 2018.

[2] W. Poewe, K. Seppi, C. M. Tanner et al., "Parkinson disease," Nature Reviews Disease Primers, vol. 3, no. 1, 2017.

[3] N. Ball, W.-P. Teo, S. Chandra, and J. Chapman, "Parkinson's disease and the environment," Frontiers in Neurology, vol. 10, p. $218,2019$. 
[4] P.-L. Zhang, Y. Chen, C.-H. Zhang, Y.-X. Wang, and P. Fernandez-Funez, "Genetics of Parkinson's disease and related disorders," Journal of medical genetics, vol. 55, no. 2, pp. 73-80, 2018.

[5] F. Brigo, R. Erro, A. Marangi, K. Bhatia, and M. Tinazzi, "Differentiating drug-induced parkinsonism from Parkinson's disease: an update on non-motor symptoms and investigations," Parkinsonism \& Related Disorders, vol. 20, no. 8, pp. 808-814, 2014.

[6] C. Marras, A. Lang, B. P. van de Warrenburg et al., "Nomenclature of genetic movement disorders: recommendations of the international Parkinson and movement disorder society task force," Movement Disorders, vol. 31, no. 4, pp. 436-457, 2016.

[7] J. J. Chen and L. Marsh, “Anxiety in Parkinson's disease: identification and management," Therapeutic Advances in Neurological Disorders, vol. 7, no. 1, pp. 52-59, 2014.

[8] L. Marsh, "Depression and Parkinson's disease: current knowledge," Current Neurology and Neuroscience Reports, vol. 13, no. 12, p. 409, 2013.

[9] L. Mah, C. Szabuniewicz, and A. J. Fiocco, "Can anxiety damage the brain?," Current Opinion in Psychiatry, vol. 29, no. 1, pp. 56-63, 2016.

[10] S. C. Trifu, A. C. Trifu, E. Aluaş, M. A. Tătaru, and R. V. Costea, "Brain changes in depression," Romanian Journal of Morphology and Embryology, vol. 61, no. 2, pp. 361-370, 2020.

[11] V. Dias, E. Junn, and M. M. Mouradian, "The role of oxidative stress in Parkinson's disease," Journal of Parkinson's Disease, vol. 3, no. 4, pp. 461-491, 2013.

[12] S. M. Jandhyala, R. Talukdar, C. Subramanyam, H. Vuyyuru, M. Sasikala, and D. Nageshwar Reddy, "Role of the normal gut microbiota," World Journal of Gastroenterology, vol. 21, no. 29, pp. 8787-8803, 2015.

[13] A. H. Tan, J. W. Hor, C. W. Chong, and S.-Y. Lim, "Probiotics for Parkinson's disease: current evidence and future directions," JGH Open, vol. 5, no. 4, pp. 414-419, 2021.

[14] M. Kechagia, D. Basoulis, S. Konstantopoulou et al., "Health benefits of probiotics: a review," International Scholarly Research Notices, vol. 2013, Article ID 481651, 7 pages, 2013.

[15] R. George Kerry, J. K. Patra, S. Gouda, Y. Park, H.-S. Shin, and G. Das, "Benefaction of probiotics for human health: a review," Journal of Food and Drug Analysis, vol. 26, no. 3, pp. 927-939, 2018.

[16] L. Magistrelli, A. Amoruso, L. Mogna et al., "Probiotics may have beneficial effects in Parkinson's disease: in vitro evidence," Frontiers in Immunology, vol. 10, p. 969, 2019.

[17] P. Gazerani, "Probiotics for Parkinson's disease," International Journal of Molecular Sciences, vol. 20, no. 17, p. 4121, 2019.

[18] O. R. Tamtaji, M. Taghizadeh, R. Daneshvar Kakhaki et al., "Clinical and metabolic response to probiotic administration in people with Parkinson's disease: a randomized, doubleblind, placebo-controlled trial," Clinical Nutrition, vol. 38, no. 3, pp. 1031-1035, 2019.

[19] M. Barichella, C. Pacchetti, C. Bolliri et al., "Probiotics and prebiotic fiber for constipation associated with Parkinson disease," Neurology, vol. 87, no. 12, pp. 1274-1280, 2016.

[20] A. H. Tan, S.-Y. Lim, K. K. Chong et al., "Probiotics for constipation in Parkinson disease," Neurology, vol. 96, pp. e772LPe782LP, 2020.

[21] R. L. Vaz, T. F. Outeiro, and J. J. Ferreira, “Zebrafish as an animal model for drug discovery in Parkinson's disease and other movement disorders: a systematic review," Frontiers in Neurology, vol. 9, p. 347, 2018.

[22] Y. Vijayanathan, F. T. Lim, S. M. Lim et al., "6-OHDAlesioned adult zebrafish as a useful Parkinson's disease model for dopaminergic neuroregeneration," Neurotoxicity Research, vol. 32, no. 3, pp. 496-508, 2017.

[23] M. E. Nunes, T. E. Muller, M. M. Braga et al., "Chronic treatment with paraquat induces brain injury, changes in antioxidant defenses system, and modulates behavioral functions in zebrafish," Molecular Neurobiology, vol. 54, no. 6, pp. 39253934, 2017.

[24] M.-A. Robea, I.-M. Balmus, A. Ciobica et al., "Parkinson's disease-induced zebrafish models: focussing on oxidative stress implications and sleep processes," Oxidative Medicine and Cellular Longevity, vol. 2020, Article ID 1370837, 15 pages, 2020.

[25] C.-W. Feng, Z.-H. Wen, S.-Y. Huang et al., "Effects of 6hydroxydopamine exposure on motor activity and biochemical expression in zebrafish (Danio rerio) larvae," Zebrafish, vol. 11, no. 3, pp. 227-239, 2014.

[26] J. W. Bortolotto, G. P. Cognato, R. R. Christoff et al., "Longterm exposure to paraquat alters behavioral parameters and dopamine levels in adult zebrafish (Danio rerio)," Zebrafish, vol. 11, no. 2, pp. 142-153, 2014.

[27] C. Milanese, J. J. Sager, Q. Bai et al., "Hypokinesia and reduced dopamine levels in zebrafish lacking $\beta \backslash$ - and $\gamma 1$-synucleins," The Journal of Biological Chemistry, vol. 287, no. 5, pp. 2971-2983, 2012.

[28] S. Martel, J. Y. Keow, and M. Ekker, "Rotenone neurotoxicity causes dopamine neuron loss in zebrafish," University of Ottawa Journal of Medicine, vol. 5, no. 2, pp. 16-21, 2015.

[29] K. M. Melo, R. Oliveira, C. K. Grisolia et al., "Short-term exposure to low doses of rotenone induces developmental, biochemical, behavioral, and histological changes in fish," Environmental Science and Pollution Research, vol. 22, no. 18, pp. 13926-13938, 2015.

[30] Y. Wang, W. Liu, J. Yang et al., "Parkinson's disease-like motor and non-motor symptoms in rotenone-treated zebrafish," Neurotoxicology, vol. 58, pp. 103-109, 2017.

[31] M. A. Robea, S. A. Strungaru, C. Lenzi, M. Nicoara, and A. Ciobica, "The importance of rotenone in generating neurological and psychiatric features in zebrafish-relevance for a Parkinson's disease model," Academy of Romanian Scientists, vol. 7, pp. 59-67, 2018.

[32] V. Bashkatova, M. Alam, A. Vanin, and W. J. Schmidt, "Chronic administration of rotenone increases levels of nitric oxide and lipid peroxidation products in rat brain," Experimental Neurology, vol. 186, no. 2, pp. 235-241, 2004.

[33] B. Reed and M. Jennings, "Guidance on the housing and care of zebrafish danio rerio," in Guidance on the housing and care of zebrafish Danio rerio, pp. 21-41, Royal Society for the Prevention of Cruelty to Animals (RSPCA), Horsham, UK, 2011.

[34] "Commission Recommendation Guidelines for the accommodation and care of animals used for experimental and other scientific purposes (notified under document number C(2007) 2525)," Official Journal of the European Union, vol. 50., 2007.

[35] "Directive 63 The protection of animals used for scientific purposes," Official Journal of the European Union, vol. 53, 2010. 
[36] S. Bretaud, S. Lee, and S. Guo, "Sensitivity of zebrafish to environmental toxins implicated in Parkinson's disease," Neurotoxicology and Teratology, vol. 26, no. 6, pp. 857-864, 2004.

[37] D. G. Valcarce, M. F. Riesco, J. M. Martínez-Vázquez, and V. Robles, "Diet supplemented with antioxidant and antiinflammatory probiotics improves sperm quality after only one spermatogenic cycle in zebrafish model," Nutrients, vol. 11, no. 4, 2019.

[38] A. M. Valentim, F. J. van Eeden, U. Strähle, and I. A. S. Olsson, "Euthanizing zebrafish legally in Europe: are the approved methods of euthanizing zebrafish appropriate to research reality and animal welfare?," EMBO Reports, vol. 17, no. 12, pp. 1688-1689, 2016.

[39] S.-A. Strungaru, G. Plavan, A. Ciobica et al., "Acute exposure to gold induces fast changes in social behavior and oxidative stress of zebrafish (Danio rerio)," Journal of Trace Elements in Medicine and Biology, vol. 50, pp. 249-256, 2018.

[40] M. A. Robea, R. Jijie, M. Nicoara et al., "Vitamin C attenuates oxidative stress and behavioral abnormalities triggered by fipronil and pyriproxyfen insecticide chronic exposure on zebrafish juvenile," Antioxidants, vol. 9, no. 10, p. 944, 2020.

[41] I.-M. Balmus, R. Lefter, A. Ciobica et al., "Preliminary biochemical description of brain oxidative stress status in irritable bowel syndrome contention-stress rat model," Medicina, vol. 55, no. 12, p. 776, 2019.

[42] Y. Jin, Z. Liu, F. Liu, Y. Ye, T. Peng, and Z. Fu, "Embryonic exposure to cadmium (II) and chromium (VI) induce behavioral alterations, oxidative stress and immunotoxicity in zebrafish (Danio rerio_)," Neurotoxicology and Teratology, vol. 48, pp. 9-17, 2015.

[43] H. Ni, L. Peng, X. Gao et al., "Effects of maduramicin on adult zebrafish (Danio rerio): acute toxicity, tissue damage and oxidative stress," Ecotoxicology and Environmental Safety, vol. 168, pp. 249-259, 2019.

[44] R. Jijie, G. Solcan, M. Nicoara, D. Micu, and S.-A. Strungaru, "Antagonistic effects in zebrafish (Danio rerio) behavior and oxidative stress induced by toxic metals and deltamethrin acute exposure," Science of the Total Environment, vol. 698, p. 134299, 2020.

[45] L. Kong, Z. Xiong, X. Song et al., "Enhanced antioxidant activity in Streptococcus thermophilus by high-level expression of superoxide dismutase," Frontiers in Microbiology, vol. 11, p. $2786,2020$.

[46] İ. Ünal, Ü. V. Üstündağ, P. S. Ateş et al., "Rotenone impairs oxidant/antioxidant balance both in brain and intestines in zebrafish," The International Journal of Neuroscience, vol. 129, no. 4, pp. 363-368, 2019.

[47] J. Blesa, I. Trigo-Damas, A. Quiroga-Varela, and V. R. Jackson-Lewis, "Oxidative stress and Parkinson's disease," Frontiers in Neuroanatomy, vol. 9, p. 91, 2015.

[48] L. Puspita, S. Y. Chung, and J.-W. Shim, "Oxidative stress and cellular pathologies in Parkinson's disease," Molecular Brain, vol. 10, no. 1, p. 53, 2017.

[49] Z. Khan and S. A. Ali, "Oxidative stress-related biomarkers in Parkinson's disease: a systematic review and meta-analysis," Iran. J. Neurol., vol. 17, no. 3, pp. 137-144, 2018.

[50] O. M. Ighodaro and O. A. Akinloye, "First line defence antioxidants-superoxide dismutase (SOD), catalase (CAT) and glutathione peroxidase (GPX): their fundamental role in the entire antioxidant defence grid," Alexandria Journal of Medicine, vol. 54, no. 4, pp. 287-293, 2018.
[51] A. N. Wang, X. W. Yi, H. F. Yu, B. Dong, and S. Y. Qiao, "Free radical scavenging activity of Lactobacillus fermentum in vitro and its antioxidative effect on growing-finishing pigs," Journal of Applied Microbiology, vol. 107, no. 4, pp. 1140-1148, 2009.

[52] D. J. Davis, E. C. Bryda, C. H. Gillespie, and A. C. Ericsson, "Microbial modulation of behavior and stress responses in zebrafish larvae," Behavioural Brain Research, vol. 311, pp. 219-227, 2016.

[53] S. H. al-Sheraji, I. Amin, A. Azlan, M. Y. Manap, and F. A. Hassan, "Effects ofBifidobacterium longumBB536 on lipid profile and histopathological changes in hypercholesterolaemic rats," Microbe, vol. 6, no. 5, pp. 661-668, 2015.

[54] H. Khotimah, S. Sumitro, and M. Widodo, "Zebrafish Parkinson's model: rotenone decrease motility, dopamine, and increase $\alpha$-synuclein aggregation and apoptosis of zebrafish brain," International Journal of PharmTech Research, vol. 8, pp. 614-621, 2015.

[55] D. Khatri and A. Juvekar, "Abrogation of locomotor impairment in a rotenone-induced Drosophila melanogaster and zebrafish model of Parkinson's disease by ellagic acid and curcumin," International Journal of Nutrition, Pharmacology, Neurological Diseases, vol. 6, no. 2, pp. 90-96, 2016.

[56] X.-J. Kong, J. Liu, J. Li et al., "Probiotics and oxytocin nasal spray as neuro-social-behavioral interventions for patients with autism spectrum disorders: a pilot randomized controlled trial protocol," Pilot and Feasibility Studies, vol. 6, no. $1,2020$.

[57] M. Hemmati-Dinarvand, S. Saedi, M. Valilo et al., "Oxidative stress and Parkinson's disease: conflict of oxidant-antioxidant systems," Neuroscience Letters, vol. 709, p. 134296, 2019.

[58] A. Masato, N. Plotegher, D. Boassa, and L. Bubacco, "Impaired dopamine metabolism in Parkinson's disease pathogenesis," Molecular Neurodegeneration, vol. 14, no. 1, p. 35, 2019.

[59] M. A. Siddiqui, J. Ahmad, N. N. Farshori et al., "Rotenoneinduced oxidative stress and apoptosis in human liver HepG2 cells," Molecular and Cellular Biochemistry, vol. 384, no. 1-2, pp. 59-69, 2013.

[60] M. C. Houser and M. G. Tansey, "The gut-brain axis: is intestinal inflammation a silent driver of Parkinson's disease pathogenesis?," NPJ Parkinson's disease, vol. 3, no. 1, 2017.

[61] G. G. Ortiz, "Gut-brain axis: role of microbiota in Parkinson's disease and multiple sclerosis," in Eat, Learn, Remember, IntechOpen, Rijeka, 2019.

[62] S. F. Santos, H. L. de Oliveira, E. S. Yamada, B. C. Neves, and A. Pereira Jr., "The gut and Parkinson's disease-a bidirectional pathway," Frontiers in Neurology, vol. 10, p. 574, 2019.

[63] Y. Bhattarai, J. Si, M. Pu et al., "Role of gut microbiota in regulating gastrointestinal dysfunction and motor symptoms in a mouse model of Parkinson's disease," Gut Microbes, vol. 13, no. 1, p. 1866974, 2021.

[64] H. B. Dodiya, C. B. Forsyth, R. M. Voigt et al., "Chronic stressinduced gut dysfunction exacerbates Parkinson's disease phenotype and pathology in a rotenone-induced mouse model of Parkinson's disease," Neurobiology of Disease, vol. 135, p. 104352, 2020.

[65] M. E. Johnson, A. Stringer, and L. Bobrovskaya, "Rotenone induces gastrointestinal pathology and microbiota alterations in a rat model of Parkinson's disease," Neurotoxicology, vol. 65, pp. 174-185, 2018.

[66] L. Bonfrate, D. M. Di Palo, G. Celano et al., "Effects of Bifidobacterium longum BB536 and Lactobacillus rhamnosus 
HN001 in IBS patients," European Journal of Clinical Investigation, vol. 50, no. 3, article e13201, 2020.

[67] M. Toscano, R. de Grandi, L. Stronati, E. de Vecchi, and L. Drago, "Effect ofLactobacillus rhamnosusHN001 andBifidobacterium longumBB536 on the healthy gut microbiota composition at phyla and species level: a preliminary study," World Journal of Gastroenterology, vol. 23, no. 15, pp. 26962704, 2017.

[68] R. Inturri, A. Stivala, P. M. Furneri, and G. Blandino, "Growth and adhesion to HT-29 cells inhibition of Gram-negatives by Bifidobacterium longum BB536 e Lactobacillus rhamnosus HN001 alone and in combination," European Review for Medical and Pharmacological Sciences, vol. 20, no. 23, pp. 49434949, 2016.

[69] R. Inturri, L. Trovato, G. L. Volti, S. Oliveri, and G. Blandino, "In vitro inhibitory activity of Bifidobacterium longum BB536 and Lactobacillus rhamnosus HN001 alone or in combination against bacterial and Candida reference strains and clinical isolates," Heliyon, vol. 5, no. 11, 2019.

[70] P. Perez-Pardo, H. B. Dodiya, P. A. Engen et al., "Gut bacterial composition in a mouse model of Parkinson's disease," Beneficial Microbes, vol. 9, no. 5, pp. 799-814, 2018.

[71] X. Yang, Y. Qian, S. Xu, Y. Song, and Q. Xiao, "Longitudinal analysis of fecal microbiome and pathologic processes in a rotenone induced mice model of Parkinson's disease," Frontiers in Aging Neuroscience, vol. 9, p. 441, 2018. 